\title{
Primary Splenic Lymphoma (PSL): A Rare Presentation of Lymphoma
}

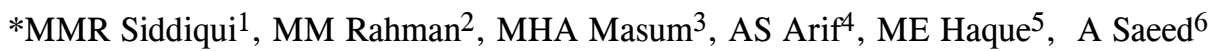

\begin{abstract}
Primary Splenic Lymphoma (PSL) is a rare neoplasm of the spleen. PSL is generally presented as B cell non-Hodgkin lymphoma. Primary involvement of the spleen by lymphoma is much less common than secondary involvement. It usually presents with nonspecific symptoms like left upper abdominal pain or discomfort due to splenomegaly, pyrexia, weight loss, night sweats and weakness. In this article, we are reporting a case of PSL in a 23 years old boy who presented with splenomegaly and non-specifc symptoms.
\end{abstract}

Key Words: Primary Splenic Lymphoma (PSL), non-Hodgkin lymphoma,

\section{Introduction}

Primary Splenic Lymphoma (PSL) is a rare neoplasm of the spleen. The spleen may be the primary site of the lymphoma or it may be a component of disseminated lymphomas. PSL comprising less than $2 \%$ of all the lymphomas and $1 \%$ of all the non-Hodgkin's lymphomas (NHL). 1,2,3 NHL comprises many subtypes, each with distinct epidemiology, etiology, and morphologic, immunophenotypic, and clinical features. Non-Hodgkin lymphoma (NHL) originates from lymphatic hematopoietic system and can be classified as B cell lymphomas (about $90 \%$ of all NHL) and $\mathrm{T}$ cell lymphomas $(10 \%$ of all NHL).4,5 Primary splenic lymphoma has been described as malignancy involving only spleen with or without splenic hilar lymph nodes with preservation of other organ systems. PSL is generally presented as B cell non-Hodgkin lymphoma. Secondary involvement of spleen is usually seen together with the involvement of abdominal lymph nodes. ${ }^{4}$

\section{Case Report}

A 23 years old boy presented with a two months history of abdominal pain, a progressive enlarging mass in the upper abdomen. He had no history of fever or night sweats. His abdominal examination revealed a large palpable spleen $(17 \mathrm{~cm})$, which was firm, tender. There was no hepatomegaly and abdominal or peripheral lymphadenopathy. The complete blood count showed $\mathrm{Hb}$ of $10 \mathrm{~g} / \mathrm{dl}$, a total count of $9,900(\mathrm{~N}-45 \%, \mathrm{~L}-50 \%, \mathrm{E}-4 \%, \mathrm{M}-1 \%)$ and a platelet count of $1,10,000$ cells/cumm. His peripheral blood smear showed normocytic normochromic anaemia and thrombocytopenia without any abnormal or atypical lymphocytes. Hid blood was negative for the malaria parasite. ICT for Malaria and Kala-azar were negative. USG of whole abdomen revealed a large splenomegaly with heterogeneous parenchyma and multiple SOL (Space occupied lesion) suggestive of primary splenic Lymphoma or granulomatous lesion. There was no splenic hilar or abdominal adenopathy. CT scan of the whole abdomen revealed huge splenomegaly with multiple SOL of variable size suggestive of splenic Lymphoma or granulomatous lesion (Fig.). There was no ascites of abdominal lymphadenopathy.

\footnotetext{
$1 *$ Dr. Md. Mahmudur Rahman Siddiqui, Associate Professor of Medicine, Anwer Khan Modern Medical College \& Hospital.

${ }^{2}$ Prof. Md. Mostafizur Rahman, Professor \& Head of Medicine, Anwer Khan Modern Medical College \& Hospital ${ }^{3}$ Dr. Md. Hasan Ali Masum, Registrar, Department of Medicine, Anwer Khan Modern Medical College \& Hospital ${ }^{4}$ Prof. Abdul Wohab Khan, Ex-Professor \& Head of Surgery, Anwer Khan Modern Medical College \& Hospital

${ }^{5}$ Prof. Md. Ehteshamul Haque, Professor \& Head, Department of Oncology, Anwer Khan Modern Medical College \& Hospital

${ }^{6}$ Dr. Atia Saeed, Assistant Professor, Department of Gastroenterology, Anwer Khan Modern Medical College \& Hospital

*Corresponding Author
}

Date of submission: 03.03.2018, Date of acceptance: 17.05.2018

AKMMC J 2018; 9(2) : 152-153 
An exploratory laparotomy was performed, which showed huge splenomegaly without any evidence of hepatomegaly or abdominal lymphadenopathy. Splenectomy was done. The spleen measured $40 \times 27 \times 17 \mathrm{~cm}$ and it weighed 4025 grams. The capsule was intact. The histopathological examination of the spleen revealed B cell type, low grade non-Hodgkin's lymphoma. The bone marrow aspiration study was normal. Immunohistochemical studies revealed features which were suggestive of splenic lymphoma of the B cell type. He did not receive any adjuvant chemotherapy post-operatively as suggested by oncologist. The patient is in complete remission two year after the diagnosis.
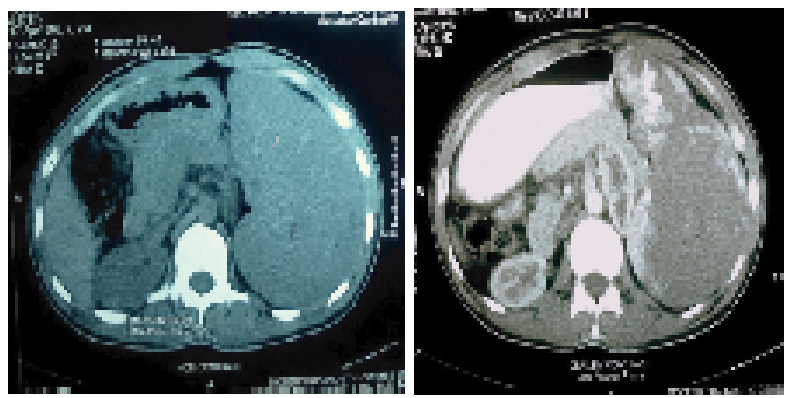

Fig: CT scan of the whole abdomen revealed huge splenomegaly with multiple SOL.

\section{Discussion}

Spleen is usually involved as part of multi systemic involvement of haematological malignancies, rather than isolated involvement. Occurrence of primary splenic NonHodgkin's Lymphoma is particularly unusual. Primary splenic tumors are rare and include angiosarcomas, lymphangiomas and malignant lymphomas. Primary involvement of the spleen by lymphoma is much less common than secondary involvement.

Das Gupta et al restrictively defined primary splenic lymphoma (PSL) as a lymphoma involving only the spleen and the splenic hilar lymph nodes, but not the liver or other sites. ${ }^{6}$ Skarin et al suggested the diagnosis of PSL for any lymphoma with splenic involvement and splenomegaly as the predominant feature. ${ }^{7}$ Kraemer et al (1984) further defined PSL as lymphoma, presenting with splenomegaly, at least bicytopenia, and in the absence of peripheral lymphadenopathy. ${ }^{8}$

PSL commonly presented with left sided abdominal pain, abdominal distension secondary to splenomegaly, fatigue, weight loss, fever and night sweating are often noted. Other symptoms usually develop secondary to direct invasion of pancreas, stomach, diaphragm and omentum. Asymptomatic Cytopenia can also be a presenting feature. The imaging techniques (USG/CT scan) are most valuable tools for diagnosis and also very helpful in excluding the involvement of abdominal lymphadenopathy and other organ involvement. ${ }^{1,9}$

The possible treatment modalities include splenectomy, local radiotherapy and systemic chemotherapy. Splenectomy is considered as the most effective therapy for PSL, because it is both a diagnostic and a therapeutic one. Local radiotherapy is considered for those in whom splenectomy is not feasible. ${ }^{1,9}$

Conflict of interest: We have no conflict of interest.

\section{References}

1. Hanumanthappa MB, Gopinathan S, Praveen N et al. Primary Splenic Lymphoma: A Rare Clinical Case Report. Journal of Clinical and Diagnostic Research. 2011 December; 5(8): 1667-1669.

2. Nuala A Healy, Conneely J B, Mahon S, et al. Primary splenic lymphoma presenting with ascites. Rare Tumours 2011;

3. Cavanna L, Artioli F, Vallisa D, et al. Primary lymphoma of the spleen. report of a case with diagnosis by fine needle guided biopsy. Heamatologica 1995; 80-241-43.

4. Rajesh K, Sravan KK, Venkateswara RK et al. Primary Follicular Lymphoma of the spleen: A Case report and literature review. IOSR Journal of Dental and Medical Sciences (IOSR-JDMS) 2015; 14(11): 31-33.

5. Coiffier B. "Monoclonal antibody as therapy for malignant lymphomas", C R Bio 2006; 329(4), 241-254.

6. Das Gupta T, Coombes B, Brasfield RD. Primary malignant neoplasms of the spleen. Surg Gynecol Obstet 1965; 120: 947-60.

7. Skarin AT, Davey FR, Moloney WC. Lymphosarcoma of the spleen: results of diagnostic splenectomy in 11 patients. Arch Intern Med. 1971; 127: 259-65.

8. Kraemer BB, Osborne BM, Butler JJ. Primary splenic presentation of malignant lymphoma and related disorders. A study of 49 cases. Cancer. 1984; 54: 1606-1619.

9. Bal Manjit S, Kaur M, Gupta N et al. Primary Splenic Hodgkin's Lymphoma: A Case Report. Research and Reviews: Journal of Medical and Health Sciences, 2014; 3(2): 38-41. 